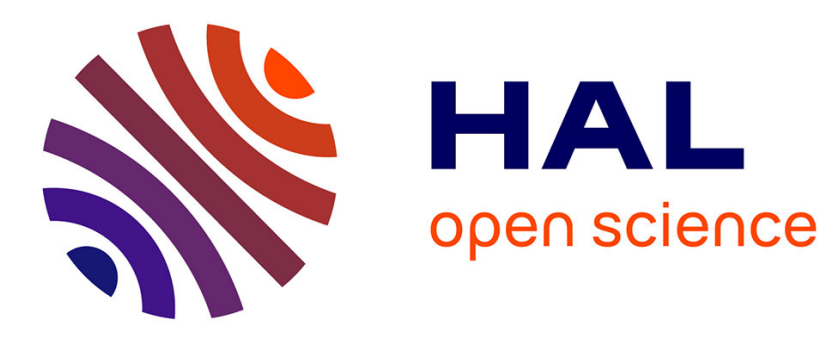

\title{
Déclaration universelle des Droits de l'homme et conflits armés : de la fragmentation à la complexité
}

\author{
Xavier Aurey
}

\section{To cite this version:}

Xavier Aurey. Déclaration universelle des Droits de l'homme et conflits armés : de la fragmentation à la complexité. Cahiers de la recherche sur les droits fondamentaux , 2009, 7, pp.49-62. hal-01350792

\section{HAL Id: hal-01350792 \\ https://hal.science/hal-01350792}

Submitted on 1 Aug 2016

HAL is a multi-disciplinary open access archive for the deposit and dissemination of scientific research documents, whether they are published or not. The documents may come from teaching and research institutions in France or abroad, or from public or private research centers.
L'archive ouverte pluridisciplinaire HAL, est destinée au dépôt et à la diffusion de documents scientifiques de niveau recherche, publiés ou non, émanant des établissements d'enseignement et de recherche français ou étrangers, des laboratoires publics ou privés.

\section{(ㅇ)(1) $\$$}

Distributed under a Creative Commons Attribution - NonCommercial - NoDerivatives| 4.0 


\title{
Déclaration universelle des Droits de l'homme et conflits armés: de la fragmentation à la complexité
}

\author{
Xavier AUREY \\ Attaché temporaire d'enseignement et de recherche à l'Université de Caen Basse-Normandie \\ Membre associé du Centre de recherche sur les droits fondamentaux et les évolutions du droit (CRDFED) \\ Doctorant à I'Université Paris II
}

I. La DUDH ou l'expression d'un simple réajustement, après la seconde guerre mondiale, du paradigme traditionnel de l'État souverain

II. La DUDH, d'une coutume à une lex generalis ou l'émergence d'un nouveau paradigme

III. DUDH et conflits armés ou une approche systémique de la société internationale

\begin{abstract}
No doubt it is true to say that international law is made for States, and not States for international law, but it is true only in the sense that the State is made for human beings, and not human beings for the State ${ }^{2}$.
\end{abstract}

Alors que nous fêtons le $60^{\mathrm{e}}$ anniversaire de la Déclaration universelle des Droits de l'homme, force est de constater que sa place dans l'ordre juridique international fait encore débat. Conçue à l'origine comme une simple déclaration d'intention, elle a acquis au fil des ans une place fondamentale qui rejoint finalement la volonté de l'un de ses pères, René Cassin ${ }^{3}$.

Adoptée au lendemain de la seconde guerre mondiale, elle trouve ses racines dans les horreurs de ce conflit $^{4}$, qui, comme le souligne justement Theodor Meron, a donné naissance au mouvement des Droits de l'homme ${ }^{5}$. Ainsi, l'après-guerre voit se concrétiser une

1. L'auteur tient à remercier tout particulièrement Jérôme Benzimra-Hazan (CRDH - Université de Paris II), Jean-Manuel Larralde et Vincent Souty (CRDFED - Université de Caen Basse-Normandie) pour leur relecture attentive et leurs précieux conseils.

2. H. Lauterpacht, The function of Law in the International Community, Oxford, Clarendon Press, 1933, p. 430-431 (relevé par P.-M. Dupuy, «The Danger of Fragmentation or Unification of the International Legal System and the International Court of Justice», International Law and Politics, vol. 31, 1999, p. 791).

3. Voir notamment R. Cassin, enregistrement réalisé dans les studios de lectures par le disque de l'Union des aveugles de guerre et diffusé au stade de Grenoble, le 17 mai 1948, à l'occasion de la célébration du $30^{\mathrm{e}}$ anniversaire de la fondation de l'Union fédérale des anciens combattants : «Je cherche, suivant la tradition de 89 , à rédiger une Déclaration internationale des Droits de l'homme, mais aussi à préparer les moyens pratiques de la faire respecter sous le contrôle de la communauté des Nations unies par-dessus les vieilles souverainetés meurtrières » (cité par M. Agi, «L'action personnelle de René Cassin ", in La Déclaration universelle des Droits de l'homme, 1948-1998. Avenir d'un idéal commun, Actes du colloque de la Sorbonne, 14-16 septembre 1998, Commission nationale consultative des Droits de l’homme (éd.), Paris, La Documentation française, 1999, p. 167).

4. Voir notamment l'intervention de S. L. Ménon, représentante de l'Inde, Assemblée générale des Nations unies, $3^{\mathrm{e}}$ session, $3^{\mathrm{e}}$ commission, $182^{\mathrm{e}}$ séance, Séance plénière, 10 décembre 1948, UN Doc. A/PV.182: «La Déclaration universelle des Droits de l’homme est née du besoin de réaffirmer ces droits après la violation qu'ils avaient subie pendant la guerre. Cette réaffirmation s'impose maintenant plus que jamais. »Voir également M. Kamto, «Rapport de séance. Deuxième séance », in La Déclaration universelle des Droits de l'homme, 1948-1998..., p. 145-149.

5. T. Meron, «International Law in the Age of Human Rights. General Course on Public International Law », RCADI, t. 301, 2003, p. 29: « The atrocities of World War II gave birth to the human rights movement, in the recognition of human rigths as a fundamental principle in the UN Charter, in the insistence on individual criminal responsibility, in the judgment of the Nuremberg Tribunal, in the promulgation of the Universal Declaration of Human Rights (1948). » 
volonté, énoncée dès $1941^{6}$, d'établir une paix durable fondée sur le respect des droits fondamentaux de l'homme. Dès le préambule de la Charte des Nations unies transparaît cet objectif: «Résolus [...] - à préserver les générations futures du fléau de la guerre qui deux fois en l'espace d'une vie humaine a infligé à l'humanité d'indicibles souffrances, - à proclamer à nouveau notre foi dans les droits fondamentaux de l'homme, dans la dignité et la valeur de la personne humaine, dans l'égalité de droits des hommes et des femmes, ainsi que des nations, grandes et petites, - à créer les conditions nécessaires au maintien de la justice et du respect des obligations nées des traités et autres sources du droit international ${ }^{7}$.»

Cette question des Droits de l'homme, expressément présente aussi aux articles 1 et 55 de la Charte ${ }^{8}$, n'y est pourtant évoquée que de manière très générale. Et si son article 56 engage les États membres des Nations unies « en vue d'atteindre les buts énoncés à l'Article 55, à agir, tant conjointement que séparément, en coopération avec l'Organisation ", les droits en question ne sont nulle part définis. C'est pourquoi, dès sa création, la Commission des Droits de l'homme eut pour mission de rédiger une Déclaration internationale qui proclamerait les droits au fondement du système mis en place par les Nations unies. Cette maïeutique juridique déboucha, en décembre 1948, sur l'adoption de la Déclaration universelle des Droits de l'homme (DUDH) ${ }^{9}$. Résolution de l'Assemblée générale des Nations unies, cette Déclaration n'en demeure pas moins un texte essentiel. Dès 1962, la Commission des Droits de l'homme précisera d'ailleurs que, de manière générale, les déclarations, instruments "solennels ", ne sont utilisées "qu'en de très rares occasions pour des questions d'importance majeure et durable, où l'on attend des membres qu'ils respectent au maximum les principes énoncés ${ }^{10}$. La Déclaration de 1948 apparait donc comme une «interprétation autorisée des principes de la Charte. Elle tire de la Charte sa pleine valeur juridique ${ }^{11}$.

Fille des horreurs de la guerre, la DUDH semble vouloir effacer en son sein toute trace de son ascendance belliqueuse. Pourtant René Cassin «mit tous ses efforts pour que l'on conservât cette trace de la guerre dans le texte ${ }^{12}$. Mais il est vrai, comme le rappelle Robert Kolb, que la mise hors la loi de la guerre par la Charte de San Francisco ne présageait pas sa prise en compte par les organes des Nations unies. En effet, « il semblait régner un consensus tacite, mais néanmoins général, que la Déclaration était une œuvre destinée au temps de paix, dont l'Organisation se portait garante ${ }^{13}$. De même, il apparaît que «parallèlement, lors de l'élaboration des Conventions de Genève de 1949, il n'a guère été question des Droits de l'homme ${ }^{14}$. Comme le rappelle le Tribunal pénal international pour l'ex-Yougoslavie (TPIY) dans sa jurisprudence Tadic de 1995, un conflit armé est caractérisé par une lutte entre forces armées organisées ${ }^{15}$. A contrario, la période de paix est celle qui ne répond pas à cette définition. Il semblait donc aisé de comprendre, en 1948, que le droit international humanitaire s'applique aux périodes de conflit armé, et le droit des Droits de l'homme aux autres cas ${ }^{16}$.

Voir également G. Cohen-Jonathan, «Rapport introductif général », in Droit international. Droits de l'homme et juridictions internationales, Bruxelles, Bruylant (Droit et justice, ${ }^{\circ}$ 55), 2004, p. 12 : «C'est pourquoi la Déclaration universelle de 1948 est si précieuse, puisque, pour la première fois, elle apportait, issue des cendres de la deuxième guerre mondiale, la réponse générale et la voie à suivre pour l'ensemble de la communauté internationale. C'était là le point de départ réel, le fondement du droit international des Droits de l'homme. »

6. Cf. F. D. Roosevelt, Discours des quatre libertés, 6 janvier 1941; Charte de l'Atlantique, 14 août 1941 ; Déclaration des Nations unies, ${ }^{\text {er }}{ }^{\text {janvier } 1942 .}$

7. Charte des Nations unies, 26 juin 1945, préambule.

8. Charte des Nations unies, article 1 : «Les buts des Nations unies sont les suivants: [... 3. Réaliser la coopération internationale en résolvant les problèmes internationaux d'ordre économique, social, intellectuel ou humanitaire, en développant et en encourageant le respect des Droits de l'homme et des libertés fondamentales pour tous, sans distinction de race, de sexe, de langue ou de religion » et article 55 : « En vue de créer les conditions de stabilité et de bien-être nécessaires pour assurer entre les nations des relations pacifiques et amicales fondées sur le respect du principe de l'égalité des droits des peuples et de leur droit à disposer d'eux-mêmes, les Nations unies favoriseront : [...] c. Le respect universel et effectif des Droits de l'homme et des libertés fondamentales pour tous, sans distinction de race, de sexe, de langue ou de religion. »

9. Déclaration universelle des Droits de l'homme, Assemblée générale des Nations unies, résolution 217 A (III), 12 décembre 1948, UN Doc. A/RES/217 (III).

10. Conseil économique et social, Rapport de la Commission des Droits de l'homme, $18^{\mathrm{e}}$ session, 19 mars-14 avril 1962, New York, Nations unies, Document des Nations unies: E/3616/Rev.1, \$105.

11. E. Decaux, «Une Déclaration au fondement de la communauté internationale », Label France, n 34, 1998. Voir également K. Azkoul, Assemblée générale des Nations unies, $3^{\mathrm{e}}$ session, $3^{\mathrm{e}}$ commission, $91^{\mathrm{e}}$ séance, 2 octobre 1948, UN Doc. A/C.3/SR.91: «Cette déclaration n'est pas que belles paroles, car aucun État ne pourrait en violer les principes sans violer en même temps les termes de la Charte. En réalité, la résolution qui invite à adopter cette déclaration est plus qu'une recommandation, car il y a déjà place dans la Charte pour une déclaration des Droits de l'homme »; J. Oraá, Human Rights in State of Exception in International Law, Oxford, Clarendon Press, 1992, p. 214: "The 1948 Universal Declaration of Human Rights contains a list of rights; but as a General Assembly resolution the instrument was not binding as such. None the less, it has been considered as an authoritative guide to the interpretation of the provisions in the Charter." A contrario, notamment, H. Lauterpacht, International Law and Human Rights, Londres, Stevens \& Sons, 1950, p. $408-417$.

12. C. Fauré, Ce que déclarer des droits veut dire: histoires, Paris, PUF, 1997, p. 212.

13. R. Kolb, «Relations entre le droit international humanitaire et les Droits de l'homme», Revue internationale de la Croix-Rouge, ${ }^{\circ}$ 831, 1998 , p. $437-447$. 14. Ibid.

15. TPIY, Chambre d'appel, affaire Le Procureur c. Dusko Tadic, alias «Dule», arrêt relatif à l'appel de la défense concernant l'exception préjudicielle d'incompétence, IT-94-1, 2 octobre 1995, $\$ 70$ : «Un conflit armé existe chaque fois qu’il y a recours à la force armée entre États ou un conflit armé prolongé entre les autorités gouvernementales et des groupes armés organisés ou entre de tels groupes au sein d'un État. » Une formule très similaire est utilisée par A. Balguy-Gallois dans son rapport annexé à l'avis consultatif de la Commission nationale consultative des Droits de l'homme sur Le respect des droits fondamentaux de la personne humaine en situation de troubles intérieurs et tensions internes au regard du droit international, septembre 2005.

16. Avec cette nuance de la seule application des principes humanitaires minimaux énoncés aux articles 3/3/3/4 communs des Conventions de Genève de 1949 dans le cadre de conflits armés internes. 
La vision de l'État moderne, issue notamment des théories volontaristes de Bodin et du réalisme de Machiavel, abondait en ce sens. Cette théorie se rencontre sur la scène internationale dès le traité de paix de Westphalie de 1648, qui posa la souveraineté et l'égalité des États comme principes fondamentaux des relations internationales. $\grave{A}$ rebours des théories de Suarez ${ }^{17}$ ou de Grotius ${ }^{18}$, Hobbes pourra ainsi limiter la matière du droit «international » aux seuls États, le droit des gens devenant alors la loi naturelle appliquée aux États ${ }^{19}$, une loi naturelle qui n'est que l'expression des rapports de force qui sévissent dans un monde privé d'une entité matérielle supérieure et toute-puissante, en l'absence, donc, d'un Léviathan universel. De même, chez Vattel, toute nation qui se gouverne elle-même sans dépendance d'un État étranger est un État souverain. Le droit des gens est donc considéré comme la Loi des souverains. La Cour permanente de justice internationale, dans la célèbre affaire du Lotus de 1927 , consacrera cette thèse en affirmant que «le droit international régit les rapports entre des États indépendants. Les règles de droit liant les États procèdent donc de la volonté de ceux-ci » ${ }^{20}$. Comme le souligne le professeur Denis Alland, ces États, «formellement égaux et foncièrement rivaux, [...] trouvent dans la confrontation de leurs puissances mutuelles une limite à leur liberté; ainsi leur situation engendre-t-elle mécaniquement des calculs de réci-procité ${ }^{21}$. Au début du $\mathrm{XX}^{\mathrm{e}}$ siècle, nous sommes encore en présence de ce modèle de la stricte indépendance souveraine des États ou «modèle westphalien ${ }^{22}$, autrement définissable comme le paradigme de la souveraineté étatique. C'est en effet à l'époque le système de représentation de la communauté internationale le plus largement accepté.

$\mathrm{Si}$, « simple continuation de la politique par d'autres moyens ${ }^{23}$, la guerre a vocation à une certaine normativité internationale, sa réglementation n'est de ce fait que le reflet de ce rapport de force. Au contraire, figure du rapport intime entre l'État et ses citoyens, les Droits de l'homme ne relevaient que de l'unique sphère interne des États ${ }^{24}$, de leur droit constitutionnel. Entre un droit de la guerre comme expression parfaite de la souveraineté des États, et une communauté internationale encore balbutiante, la DUDH ne semblait donc pouvoir «guider» les hommes et les nations que dans les périodes où le droit des conflits armés ne trouvait pas à s'appliquer.

Mais en relisant l'arrêt Tadic précité, on s'aperçoit que cette Déclaration a joué en pratique un rôle beaucoup plus global, et notamment sur le développement du droit des conflits armés: «[L]e développement et la propagation rapides dans la communauté internationale des doctrines des Droits de l'homme, en particulier après l'adoption de la Déclaration universelle des Droits de l'homme en 1948, ont apporté des changements significatifs au droit international, en particulier dans l'approche des problèmes qui assaillent la communauté mondiale. Une approche axée sur la souveraineté de l'État a été progressivement supplantée par une approche axée sur les Droits de l'homme. Progressivement, la maxime du droit romain hominum causa omne jus constitutum est (tout droit est créé au bénéfice des êtres humains) a acquis également un solide point d'ancrage dans la communauté internationale ${ }^{25}$.

Se fondant notamment sur ce constat, le TPIY en conclut que «dans le domaine des conflits armés, la distinction entre conflits entre États et guerres civiles perd de sa valeur en ce qui concerne les personnes ${ }^{26}$. Le droit international continue, toujours selon la chambre d'appel du tribunal, à sauvegarder «les intérêts légitimes des États », mais il «doit progressivement assurer la protection des êtres humains ${ }^{27}$. Ce remplacement progressif d'une «approche axée sur la souveraineté de l'État» par une «approche axée sur les Droits de l'homme ${ }^{28}$ cache en réalité plus qu'une simple évolution du droit international $^{29}$. Nous voudrions en effet démontrer ici que la

17. Inspiré par la théologie chrétienne, Suarez fait du droit des gens un droit positif humain, résultant de ce que chaque peuple estime devoir être l'application du droit naturel divin. Ce droit volontaire doit cependant toujours être conforme au droit naturel immuable qui reste une norme supérieure. Suarez, dans ses écrits, parle également de la nécessaire existence d'une société des Nations.

18. S'inspirant des penseurs de la seconde scholastique espagnole, Grotius basa ses théories du droit des gens sur l'existence d'une société universelle qui a pour fondement la nature et la raison. Le droit des gens, intimement lié au droit naturel, n'est pas une création des États. C'est un ensemble de règles objectives, inhérentes à la nature, c'est-à-dire à la solidarité des peuples. Les États ne le font pas naître par un acte de volonté, mais ils le découvrent par un acte d'intelligence. Il existe à côté de ce droit idéal normatif, un droit constructif, issu de la volonté des États, qui se doit de respecter le droit naturel.

19. T. Hobbes, De Cive, II, XIV, IV.

20. CPJI, Affaire du Lotus (France c. Turquie), 7 septembre 1927, série A, nº 10, p. 18.

21. D. Alland, «Droit international public», in Dictionnaire de culture juridique, D. Alland et S. Rials (dir.), Paris, PUF, 2003, p. 498.

22. Cf. A. Cassese, Le droit international dans un monde divisé, Paris, Berget-Levrault, 1986, p. 345 sq.

23. C. von Clausewitz, De la Guerre, Paris, Perrin, 1999, p. 46.

24. Habeas Corpus, Déclaration d'indépendance des États-Unis, Déclaration des Droits de l’homme et du citoyen française... Voir R. Kolb, « Relations entre le droit international humanitaire et les Droits de l'homme», p. 437: «Les Droits de l'homme ont trait à l'organisation du pouvoir étatique face à l'individu. Ils sont le produit des théories du siècle des Lumières sur l'État et ont tout naturellement trouvé leur expression dans le droit constitutionnel interne.»

25. TPIY, Chambre d'appel, Le Procureur c. Dusko Tadic, alias « Dule», arrêt relatif à l'appel de la défense concernant l'exception préjudicielle d'incompétence, IT-94-1, 2 octobre 1995, $\$ 97$.

26. Ibid.

27. Ibid.

28. Voir note 15 : extrait de l'arrêt Tadic reproduit.

29. Voir également L. Despouy, Sous-Commission de la lutte contre les mesures discriminatoires et de la protection des minorités, Dixième rapport annuel et liste d'États qui, depuis le $1^{\text {er }}$ janvier 1985, ont proclamé, prorogé ou abrogé un état d'exception, 23 juin 1997, UN Doc. E/CN.4/Sub.2/1997/19, \$1: « Parmi les grands changements que l'on a observés tout au long de ce siècle, il ne fait aucun doute que l'un des plus importants et des plus révolutionnaires s'est 
DUDH est le point d'ancrage, non pas d'une évolution quasi linéaire du droit international comme semble le sous-entendre le TPIY, mais bien d'une "révolution", d'un «changement de paradigme», selon des mots que nous empruntons à Thomas Samuel Kuhn ${ }^{30}$. Ce changement radical se présente sous la forme d'un cheminement ternaire construit autour, premièrement, d'une prise de conscience d'une anomalie non résolue par le paradigme de la souveraineté étatique; ensuite, d'une instabilité théorique et pratique liée aux efforts pour résoudre cette crise, notamment par des tentatives de réajustement du modèle initial; et enfin de la mise en place d'un nouveau cadre conceptuel.

Dans le monde du droit international depuis la fin de la première guerre mondiale, l'objectif était de parvenir à une paix universelle. En se fondant sur le strict modèle de la souveraineté étatique, la Société des Nations va tenter d'arriver à cette fin. Mais nazisme et fascisme sont les premiers éléments de cette crise qui va mettre à mal ce modèle ${ }^{31}$. Ainsi, la seconde guerre mondiale semble démontrer que le paradigme en vigueur à l'époque n'était pas adapté à la résolution de cette anomalie du système international, au sens kuhnien du terme. C'est cette prise de conscience qui va notamment mener à la création des Nations unies et à la rédaction de la Déclaration universelle des Droits de l'homme.

Face à cette crise du système international, la première étape, symbolisée par le processus d'adoption de la DUDH, consistera en un réajustement du modèle traditionnel de souveraineté (I). Mais le développement des Nations unies et l'émergence de nouvelles formes de conflits vont de nouveau mettre à mal cette interprétation et voir l'émergence d'un possible nouveau paradigme (II), favorisant ainsi une approche systémique de la société internationale (III).

\section{La DUDH ou l'expression d'un simple réajustement, après la seconde guerre mondiale, du paradigme traditionnel de l'État souverain}

Fondé sur un maillage de rapports réciproques, le droit international du début du $\mathrm{XX}^{\mathrm{e}}$ siècle conserve le seul consentement des États comme base de son carac- tère obligatoire. Au sortir de la première guerre mondiale, les États occidentaux se donnent pour objectif d'établir une paix universelle et durable par la constitution d'une entité internationale chargée de la préserver. Mais tuée dans l'œuf par l'absence de quelques puissances majeures et d'obligations réelles pour les États, la SDN ne put finalement pas empêcher la seconde guerre mondiale. Au regard du paradigme de la souveraineté étatique et de son objectif de paix universelle, cet échec ne peut être considéré que comme une crise majeure. Toute la question est alors de comprendre la réaction qui a cherché à y répondre.

Comme nous le soulignions en introduction, ce conflit majeur est à la source des Nations unies et de la DUDH. Les procès de Nuremberg et de Tokyo ont profondément marqué la communauté internationale en révélant au monde l'horreur des crimes commis par les nazis et leurs alliés. Et plus que la facette purement militaire, ce sont les exactions des régimes de Hitler, de Mussolini ou de Hirohito qui ont manifestement servi de fil conducteur aux rédacteurs de la Déclaration de 1948. Johanes Morsink précise d'ailleurs qu'à la troisième Assemblée générale des Nations unies, «[a]ll the delegations generally agreed that the pattern of gross human rights abuses which occurred during World War II was the major impulse behind the drafting of the Declaration ${ }^{32}$. Cet auteur s'attache à détailler les éléments du processus d'élaboration de la DUDH liés à l'expérience du nazisme et du fascisme et met en lumière l'influence capitale de la guerre sur l'avenir des Droits de l'homme au niveau international.

Cette guerre a, de la sorte, internationalisé les Droits de l'homme en tant que discipline. Auparavant de la seule compétence interne des États, les Droits de l'homme se trouvent désormais portés par un mouvement sans précédent $^{33}$. Dès 1951, dans un cours à l'Académie de droit international de La Haye, René Cassin souligne cette évolution fondamentale: "Ainsi, la rupture s'est faite sur le problème vital des Droits de l'homme. Et l'un des enjeux essentiels de la tragédie qui a suivi a été de savoir si l'homme devait rester ou redevenir une chose ou si, pouvant être individuellement victime ou coupable au regard du droit international, il ne devait pas voir reconnaître sa qualité de "personne du droit international", soumise comme telle à des obligations, mais ayant aussi vocation juridique à des protections et à des garanties du droit des gens ${ }^{34}$.»

produit dans le domaine des Droits de l'homme, où s'est opéré un déplacement progressif d'une conception traditionnelle centrée sur l'État protagoniste vers une conception nouvelle axée davantage sur l'être humain que sur les structures exerçant le pouvoir. »

30. T. S. Kuhn, La structure des révolutions scientifiques, Paris, Flammarion, 1972. Selon cet auteur, toute science a pour point d'ancrage un paradigme. Ce paradigme est un modèle cohérent reposant sur un système d'hypothèses. C'est donc une superstructure (au sens marxien du terme, à savoir donc l'ensemble des idées d'une société, c'est-à-dire ses productions non matérielles) adaptée à un certain état du développement scientifique. Tant que les scientifiques ne sont pas confrontés à une anomalie que ne peut résoudre leur modèle, le paradigme demeure. Sinon, nous nous retrouvons face à une crise. Dans la plupart des cas, les scientifiques élaboreront de nouvelles versions et des remaniements adéquats de leur théorie afin d'éliminer tout conflit apparent. Mais si la crise demeure, il y a remise en cause du modèle et émergence d'un nouveau paradigme présentant une réponse possible à la crise.

31. Cf. également M. Delmas-Marty, Le flou du droit, Paris, PUF (Quadrige), 2004, p. 347. Cette auteure voit dans la seconde guerre mondiale « un tournant, une mutation».

32. J. Morsink, «World War Two and the Universal Declaration», Human Rights Quarterly, vol. 15, 1993, p. 357.

33. Cf. notamment la Déclaration américaine des droits et devoirs de l'homme (dite Déclaration de Bogotá), Organisation des États américains, 2 mai 1948; Convention pour la prévention et la répression du crime de génocide, Assemblée générale des Nations unies, résolution 260 A (III), 9 décembre 1948.

34. R. Cassin, « La Déclaration universelle et la mise en œuvre des Droits de l’homme», RCADI, t. 79, 1951-II, p. 242. 
La Déclaration universelle marque ainsi le dépassement, par les États, de la seule préservation de leurs intérêts légitimes ${ }^{35}$ et transfigure le droit international luimême. Les années de guerre précédant son adoption avaient vu des millions de morts parce que les principes de respect des Droits de l'homme avaient été bafoués et que le droit international d'alors n'avait pas su y répondre. Ainsi, René Cassin pouvait-il demander que « les Nations unies proclament une fois de plus à la face de l'humanité les principes qui ont failli disparaître et qu'elles rejettent catégoriquement l'abominable doctrine fasciste ${ }^{36}$. Dans l'idée des rédacteurs de la Déclaration et des représentants des États membres, les crimes et agressions des régimes nazi et fasciste étaient nés et avaient prospéré sur la négation des Droits de l'homme dans leur propre pays ${ }^{37}$. Le maintien de la paix au niveau international semblait donc conditionné par une proclamation préalable des principes fondamentaux inhérents à l'homme, seuls à même d'éviter tout retour de ces doctrines. Pour garantir une paix mondiale durable, les États acceptaient de suivre un guide international, une "simple déclaration-manifeste ${ }^{38}$, conditionnant leur action nationale. La communauté internationale n'était toutefois pas encore au stade de possibles normes obligatoires régissant la matière ${ }^{39}$.

C'est en ce sens que l'on comprend que le paradigme de la souveraineté étatique n'est ici pas remis en cause. Depuis que Hobbes a fondé la légitimité de la souveraineté du Léviathan sur sa capacité à empêcher les individus de mourir de mort violente, les choses n'ont que peu évolué. En 1948, malgré la volonté de certains membres de la Commission des Droits de l'homme, ce paradigme ne se trouve que réajusté et ses fondements ne sont en aucun cas renversés. Ainsi, la Charte des Nations unies et la Déclaration de 1948, en oubliant le droit des conflits armés, ont entériné pour un temps la manifestation principale de ce modèle westphalien qu'est la dichotomie entre Droits de l'homme et droit des conflits armés.
Une stricte application du paradigme de la souveraineté étatique ne pouvait en effet mener qu'à une stricte séparation de ces deux corpus juridiques. Tout semblait devoir les différencier: sphère interne face à la scène internationale, temps de paix face au temps de guerre...

Appelée parfois «différentialiste», «autonomiste» ou « séparatiste», cette théorie se fonde sur les divergences structurelles de ces deux corpus, liées à l'histoire, à leurs moyens de mise en œuvre et à leurs champs d'application, pour affirmer le caractère infranchissable du fossé qui les sépare ${ }^{40}$. Comme le souligne Amna Guellali, analysant ce courant de pensée, «les Droits de l'homme visent à créer un modèle d'harmonie et de cohésion dans la société humaine, tandis que le droit humanitaire s'applique dans la réglementation des situations d'extrême violence ${ }^{41}$.

Et pourtant, les négociations qui ont eu lieu entre 1947 et 1948 dans l'enceinte des Nations unies auraient pu influencer, même indirectement, celles qui, se déroulant peu de temps après à Genève, ont abouti aux quatre Conventions de 1949. Plusieurs projets de préambule à ces Conventions se référaient d'ailleurs directement à la Déclaration de $1948^{42}$, mais «[f] aute d'être parvenues à un accord, les Conventions de 1949 sont restées sans préambule ${ }^{43}$.

Dans son ouvrage sur les Droits de l'homme et le droit humanitaire, René Provost voit tout de même une influence de la DUDH sur le texte des Conventions de Genève: "in the provisions prohibiting discriminations (Arts. 12/12/16/27) [or] in other provisions dealing with torture, cruel, unusual and degrading treatment or punishment, arbitrary arrest or detention, and due process $"{ }^{44}$. René-Jean Wilhelm se montre, pour sa part, beaucoup plus nuancé. Il ne relève que deux règles des Conventions de Genève « où l'on sente implicitement l'influence de la Déclaration universelle des Droits de l'homme et de la Charte des Nations unies ", à savoir, "celle qui proscrit

35. Cf. A. Migliazza, «L'évolution de la réglementation de la guerre à la lumière de la sauvegarde des Droits de l'homme », RCADI, t. 137, 1972-III, p. 194: «Les Droits de l'homme ont mis en lumière le but fondamental poursuivi par le droit de la guerre, soit la protection et la sauvegarde des valeurs humaines : par cela, ils ont mis l'accent sur la protection des individus impliqués dans des conflits tandis que selon la doctrine classique, le droit de la guerre visait plutôt la protection de groupes humains et d'intérêts étatiques. »

36. Assemblée générale des Nations unies, $3^{\mathrm{e}}$ session, $3^{\mathrm{e}}$ commission, $96^{\mathrm{e}}$ séance, 7 octobre 1948 , UN Doc. A/C.3/SR.96.

37. Déclaration universelle des Droits de l’homme, préambule, $3^{\mathrm{e}}$ considérant: «Considérant que la méconnaissance et le mépris des Droits de l'homme ont conduit à des actes de barbarie qui révoltent la conscience de l'humanité. » Voir également R. Cassin, Note au Quai d'Orsay, 27 février 1947: « Dès le début de la guerre, nous avons compris que c'était la guerre des Droits de l'homme. Nous nous sommes alors donnés pour but de notre victoire de remettre au premier plan les droits de l'individu. Car il ne faut pas oublier qu'Hitler a commencé par écraser l'être humain chez lui avant de déclencher ses agressions étrangères"; Secrétaire général des Nations unies, Rapport sur le respect des Droits de l'homme en période de conflit armé, UN Doc. A/7720, novembre 1969, $\$ 16$ : «La deuxième guerre mondiale a montré de façon concluante la relation étroite qui existe entre l'attitude révoltante d'un gouvernement à l'égard de ses propres ressortissants et l'agression qu'il perpètre contre d'autres nations et, par conséquent, entre le respect des Droits de l'homme et le maintien de la paix. »

38. Cette idée d'une simple déclaration-manifeste a été officiellement proposée le 28 janvier 1947 par la représentante des États-Unis (V.E./CN.4 4/4).

39. Cf. C. Ortiz (Mexique), Assemblée générale des Nations unies, $3^{\mathrm{e}}$ session, $3^{\mathrm{e}}$ commission, $90^{\mathrm{e}}$ séance, $1^{\mathrm{er}}$ octobre 1948 , UN Doc. A/C.3/SR.90: "Cette déclaration n'entraînera pas d'obligations d'ordre juridique, mais la valeur de ce document n'en sera pas diminuée. Elle définira les Droits de l'homme que les États se sont engagés à reconnaître et servira de critère pour guider et pour stimuler ces États. À l'heure actuelle, il serait difficile d'aller plus loin. »

40. Cf. notamment R. Abi-Saab, «Human Rights and Humanitarian Law in Internal Conflicts», in Human Rights and Humanitarian Law. The Quest for Universality, D. Warner (éd.), La Haye, Martin Nijhoff, 1997, p. 107 ; Y. Dinstein, «The International Law of Inter-State Wars and Human Rights », Israel Yearbook on Human Rights, vol. 7, 1977, p. 139-153; H. Meyrovitz, «Le droit de la guerre et les Droits de l'homme », RDP, vol. 88, 1972, p. 1059-1104.

41. A. Guellali, «Lex specialis, droit international humanitaire et Droits de l'homme : leur interaction dans les nouveaux conflits armés », RGDIP, vol. 111, $\mathrm{n}^{\circ} 3,2007, \mathrm{p} .540$.

42. Cf. Actes de la Conférence diplomatique de Genève de 1949, Berne, Département politique fédéral, 1949, vol. II A, p. 761-766; vol. III, p. 96-100.

43. S. Junod, «Les Droits de l'homme et le protocole II ", RICR, vol. 739, 1983, p. 254-255.

44. R. Provost, International Human Rights and Humanitarian Law, Cambridge, Cambridge University Press, 2002, p. 6. 
les atteintes à la dignité des personnes, et notamment les traitements humiliants et dégradants » et, «la règle de l'article 3 qui interdit toute discrimination $"{ }^{45}$ qui, selon lui, serait directement inspirée de l'article 2 de la DUDH.

Les rédacteurs des Conventions de Genève se sont donc au final très peu référés aux principes fondamentaux proclamés moins d'un an auparavant. Même dans le cadre de l'article 3 commun touchant spécifiquement les conflits armés internes aux États, l'inspiration a davantage été trouvée dans la tradition humanitaire et l'esprit même de ces quatre Conventions de Genève ${ }^{46}$ que dans les Droits de l'homme. C'était pourtant, à l'époque, le cadre le plus propice pour une influence de la DUDH: relevant de la sphère interne des États, la réglementation des conflits armés non internationaux partage avec elle le même souci de protection des individus contre leur propre État.

Au-delà de cette question de l'influence de la DUDH sur le droit des conflits armés s'est posée dès 1948 celle, beaucoup plus conflictuelle, d'une possible applicabilité aux rapports de guerre des droits ainsi proclamés. L'argumentaire d'Henri Meyrowitz ${ }^{47}$ est, sur ce point, fort intéressant. Partisan d'une vision différentialiste, il réfute les théories qui, voyant dans la Déclaration une réaction aux crimes contre l'humanité et aux crimes de guerre commis pendant la seconde guerre mondiale, feraient de ce texte un élément commun aux Droits de l'homme et au droit humanitaire. Il affirme ainsi : «Son inapplicabilité à ces rapports [de guerre] résulte tant des présupposés philosophiques, de l'objet et du but du document que du contenu des Droits de l'homme qu'il proclame. Parce que les auteurs de la Déclaration ont considéré le respect des Droits de l'homme à l'intérieur de chaque État comme une condition capitale de la sauvegarde de la paix, c'est dans le seul cadre de la paix qu'ils ont envisagé ces droits et qu'ils se sont attachés à les définir [...]. Malgré la tournure "naturaliste" de certaines formules, les rapports auxquels la Déclaration est destinée à s'appliquer sont essentiellement des rapports "civils", c'est-à-dire les rapports de l'individu-citoyen avec la Cité, et, principalement, avec sa Citét ${ }^{48}$.»

Replacée dans le contexte de 1948, cette analyse ne peut qu'emporter l'adhésion. En effet, dans l'esprit de la majorité de ses rédacteurs, la Déclaration visait à créer les conditions d'une paix universelle par la promotion de «l'établissement d'un droit constitutionnel minimum uniforme, procédant d'une conception commune des Droits de l'homme et des libertés fondamentales ${ }^{49}$.

La guerre mise hors la loi, le système des Nations unies se concentra donc dans un premier temps sur l'évolution interne des États. Même si certains auteurs voient a posteriori dans la DUDH le moteur d'une évolution, dès 1949, du droit des conflits armés ${ }^{50}$, il nous semble anachronique, au regard du système international de l'époque, d'en arriver à une telle conclusion. Sans renier les influences réciproques observables, dès cette date, entre les deux corpus, la véritable évolution, ou révolution, viendra, quelques années plus tard, de la réappropriation par les Nations unies d'une partie du droit des conflits armés. Ce mouvement au sein des Nations unies nous paraît propre à faire de la DUDH le point de départ d'un nouveau modèle pour le droit international.

\section{La DUDH, d'une coutume à une lex generalis ou l'émergence d'un nouveau paradigme}

À la $108^{\mathrm{e}}$ séance de la $3^{\mathrm{e}}$ commission de l'Assemblée générale des Nations unies chargée de la rédaction de la Déclaration, le représentant de la Belgique, Fernand Dehousse, a prononcé une déclaration sur la portée juridique future de ce texte ${ }^{51}$. Il y fait la distinction, reprise en 1953 par Boris Mirkine-Guétzévitch ${ }^{52}$, entre les articles qui ne feront que reprendre des règles figurant déjà dans le droit international coutumier, et ceux qui exprimeront des règles nouvelles sur la scène internationale. Concernant la première série de normes, M. Dehousse

45. R.-J. Wilhelm, « Problèmes relatifs à la protection de la personne humaine par le droit international dans les conflits armés ne présentant pas un caractère international», RCADI, t. 137, 1972-III, p. 366.

46. Ibid., p. 365-366: "Ainsi la règle centrale de cette disposition, à savoir le traitement humain qui doit être accordé aux personnes ne participant pas directement aux hostilités ou à celles qui ont été mises hors de combat, tient directement de la tradition des Conventions de Genève et de celles de La Haye : la notion de "traitement humain" se trouve dans le "Code des prisonniers de guerre" de 1929, dans le Règlement de La Haye de 1907 ainsi que dans l'expression des "lois de l'humanité" contenues dans une des clauses du préambule de la IV Convention de La Haye de 1907 (clause dite de Martens [...]). Les autres règles de l'article 3 sont également inspirées des Conventions de Genève, qu'il s'agisse de l'interdiction de la prise d'otages (IV ${ }^{\mathrm{e}}$ Convention, art. 34), de l'obligation de recueillir et de soigner les blessés et les malades ( ${ }^{\mathrm{re}}$ Convention, art. 40), qu'il s'agisse enfin, de l'obligation de ne pas prononcer de condamnation ou d'effectuer des exécutions sans un jugement préalable rendu par un tribunal régulièrement constitué et assorti des garanties judiciaires nécessaires (III ${ }^{\mathrm{e}}$ Convention, art. 84). »

47. Cf. H. Meyrowitz, «Le droit de la guerre et les Droits de l’homme», RDP, vol. 88, n 5, 1972, p. 1059-1104.

48. Ibid., p. 1082.

49. Ibid., p. 1083 .

50. T. Meron, «The Humanization of Humanitarian Law», American Journal of International Law, vol. 94, $\mathrm{n}^{\circ} 2,2000$, p. 245 : «Despite these early signals [Lieber Code and Martens Clause], it is the Universal Declaration of Human Rights and other post-Charter human rights treaties and declarations that explain the focus of the Geneva Conventions and Additional Protocols on individuals and populations. In matter such as the prohibition of torture and cruel, inhuman, or degrading treatment and punishment, arbitrary arrest and detention, and discrimination, as well as the guarantees of due process of law, human rights have exercised vast influence on instruments of international humanitarian law, producing a large measure of parallelism between norms, and a growing measure of convergence in their personal and territorial applicability.»

51. Cf. F. Dehousse, Assemblée générale des Nations unies, $3^{\mathrm{e}}$ session, $3^{\mathrm{e}}$ commission, $108^{\mathrm{e}}$ séance, 20 octobre 1948, UN Doc. A/C.3/SR.108.

52. B. Mirkine-Guétzévitch, «Quelques problèmes de la mise en œuvre de la Déclaration universelle des Droits de l'homme», RCADI, vol. 83, 1953-III, p. 310-311. 
peut en conclure que «le fait de les transcrire dans une déclaration internationale ne peut priver ces règles de la valeur obligatoire qu'elles possèdent déjà » ${ }^{53}$ au titre du droit coutumier. En effet, comme le soulignera quelques années plus tard la Cour internationale de justice (CIJ) dans son arrêt Activités militaires et paramilitaires au Nicaragua et contre celui-ci, « même si deux normes provenant des deux sources du droit international apparaissent identiques par leur contenu, [...] ces normes conservent une existence distincte. Il en est ainsi du point de vue de leur applicabilité ${ }^{54}$. Cette affirmation, adoptée sur la question d'un conflit entre droit conventionnel et droit coutumier, n'en trouve pas moins, a fortiori, à s'appliquer entre droit déclaratoire et droit coutumier.

D'un autre côté, concernant l'inscription de règles non coutumières dans une résolution de l'Assemblée générale, la CIJ a pu rappeler que ces résolutions peuvent « fournir des éléments de preuve importants pour établir l'existence d'une règle ou l'émergence d'une opinio juris ${ }^{55}$. Ainsi, de manière quasi prédictive, F. Dehousse pouvait-il conclure que, de par son statut juridique, la Déclaration de 1948 allait créer «un commencement d'obligation pour les États membres des Nations unies » ${ }^{56}$. Si la Cour a également précisé que « des résolutions successives peuvent illustrer l'évolution progressive de l'opinio juris nécessaire à l'établissement d'une règle nouvelle » ${ }^{57}$, il faut donc en conclure, avec Sean MacBride, que la Déclaration universelle des Droits de l'homme, tant de fois réaffirmée et citée dans des décisions de l'Assemblée générale et dans les conventions internationales, «can certainly be regarded as enunciating "the principles of the law of nations, derived from the usages established among civilized peoples from the laws of humanity and from the dictates of the public conscience" " ${ }^{58}$. Il semble en effet que le caractère coutumier de la DUDH ne fasse aujourd'hui plus guère débat, au moins pour son application en temps de paix.
Mais, en 1967, un premier document du Conseil de sécurité des Nations unies associe explicitement les Droits de l'homme au droit des conflits armés dans une résolution relative au conflit israélo-arabe. Ce texte affirme que "les Droits de l'homme essentiels et inaliénables doivent être respectés même dans les vicissitudes de la guerre ${ }^{59}$. Corrélativement, dès 1968, après avoir demandé à Israël de « respecter et appliquer dans les territoires occupés la Déclaration universelle des Droits de l'homme et les Conventions de Genève de $1949{ }^{60}$, la Conférence internationale des Droits de l'homme tenue à Téhéran concluait ses travaux en exposant que «la Déclaration universelle des Droits de l'homme exprime la conception commune qu'ont les peuples du monde entier des droits inaliénables et inviolables inhérents à tous les membres de la famille humaine et constitue une obligation pour les membres de la communauté internationale ${ }^{61}$.

Par une simple subrogation de termes, le secrétaire général des Nations unies, dans son deuxième rapport sur Le respect des Droits de l'homme en période de conflit armé, a pu en déduire "que le respect en temps de paix, ainsi qu'en temps de conflit armé, des droits énoncés dans la Déclaration universelle des Droits de l'homme constitue maintenant une obligation importante pour les États, y compris les États engagés dans un conflit armé » ${ }^{62}$. Il apparaît ainsi qu'en 1970, M. U Thant considérait que la Déclaration avait acquis une valeur coutumière aussi bien en période de paix qu'en période de conflit armé. C'était là la première étape d'une réappropriation, par les Nations unies, de cette question de la réglementation des conflits armés.

Ainsi, par sa résolution 2675 (XXV) du 9 décembre $1970{ }^{63}$ accueillant le second rapport du secrétaire général cité ci-dessus, l'Assemblée générale des Nations unies a affirmé «les principes fondamentaux [...] touchant la protection des populations civiles en période de conflit armé ${ }^{64}$ au sein desquels elle inclue, en première

53. Cf. Fernand Dehousse, Assemblée générale des Nations unies, $3^{\mathrm{e}}$ session, $3^{\mathrm{e}}$ commission, $108^{\mathrm{e}}$ séance, 20 octobre 1948, UN Doc. A/C.3/SR.108.

54. CIJ, Activités militaires et paramilitaires au Nicaragua et contre celui-ci (Nicaragua c. États-Unis d’Amérique), fond, arrêt, 27 juin 1986, CIJ. Rec., 1986, \$174

55. CIJ, Licéité de la menace ou de l’emploi d’armes nucléaires, avis consultatif, 8 juillet 1996, CIJ Rec., 1996, \$70, p. 226 sq.

56. F. Dehousse, Assemblée générale des Nations unies, $3^{\mathrm{e}}$ session, $3^{\mathrm{e}}$ commission, $108^{\mathrm{e}}$ séance, 20 octobre 1948, UN Doc. A/C.3/SR.108.

57. CIJ, Licéité de la menace ou de l'emploi d’armes nucléaires, avis consultatif, 8 juillet 1996, CIJ Rec., 1996, \$ 70, p. 226 sq.

58. S. MacBride, «Human Rights in Armed Conflict. The Inter-Relationship between the Humanitarian Laws and the Law of Human Rights», Revue de droit pénal militaire et de droit de la guerre, vol. IX, $\mathrm{n}^{\circ}$ 1, 1970, p. 378. Cf. également Assemblée générale des Nations unies, Le respect des Droits de l’homme en période de conflit armé, Deuxième rapport du secrétaire général, 18 septembre 1970, UN Doc. A/8052, Annexe I : règles générales concernant le respect des Droits de l'homme et leur application aux conflits armés.

59. Conseil de sécurité des Nations unies, Résolution 237 (1967). La situation au Moyen-Orient, 14 juin 1967, UN Doc. S/RES/237 (1967). Nous soulignons. 60. Conférence des Nations unies de Téhéran, Résolution I: Respect et application des Droits de l'homme dans les territoires occupés, 12 mai 1968 , UN Doc. A/Conf.32/41, p. 4 .

61. Conférence des Nations unies de Téhéran, Proclamation de Téhéran. Actes finaux de la Conférence internationale sur les Droits de l’homme, 13 mai 1968, UN Doc. A/CONF.32/41, \$2. Nous soulignons.

62. Assemblée générale des Nations unies, Le respect des Droits de l'homme en période de conflit armé, Deuxième rapport du secrétaire général, 18 septembre 1970, UN Doc. A/8052, Annexe I: règles générales concernant le respect des Droits de l’homme et leur application aux conflits armés, \$5. Une approche toujours réaffirmée trente ans plus tard par le secrétaire général des Nations unies: Commission des Droits de l’homme des Nations unies, Sous-Commission de la lutte contre les mesures discriminatoires et de la protection des minorités, Règles humanitaires minimales, Rapport analytique soumis par le secrétaire général en application de la résolution 1997/21 de la Commission des Droits de l’homme, UN Doc. E/CN.4/1998/87, 5 janvier 1998, \$ 48, p. 14: «Il est largement accepté que la Déclaration universelle des Droits de l'homme, bien qu'il ne s'agisse pas d'un traité à proprement parler, crée des obligations pour tous les États membres de l'ONU. Mais, surtout, la Déclaration universelle stipule que les Droits de l'homme sont "inaliénables" et que les êtres humains "naissent libres et égaux en dignité et en droits", d’où il découle que nous possédons ces droits, que le pays dans lequel nous vivons soit en guerre ou pas. "

63. Assemblée générale des Nations unies, Résolution 2675 (XXV). Principes fondamentaux touchant la protection des populations civiles en période de conflit armé, 9 décembre 1970, UN Doc. A/RES/2675 (XXV).

64. Ibid. 
position, «[1] es droits fondamentaux de l'homme, tels qu'ils sont acceptés en droit international et énoncés dans des instruments internationaux» et qui, donc, "demeurent pleinement applicables en cas de conflit armé ${ }^{65}$. Cette résolution, bien que ne citant pas expressément la DUDH, est dans les termes très proche desdites conclusions du secrétaire général. Ainsi, l'ensemble coutumier des droits fondamentaux de la personne, « codifié » par la DUDH, serait pleinement applicable aux civils en période de conflit armé ${ }^{66}$. Outre que ces dispositions, selon l'Assemblée générale, ne semblent $a$ priori pas viser les combattants, il nous paraît inopportun de parler ici de « confusionisme ${ }^{67}$, pour reprendre les termes d'Henri Meyrowitz. Selon cet auteur, les États arabes auraient profité de la Conférence de Téhéran pour lier politiquement les Droits de l'homme au droit international humanitaire. Ainsi cette Conférence aurait fourni «à point nommé aux États arabes l'occasion de porter devant l'opinion mondiale les accusations lancées contre la conduite d'Israël dans les territoires occupés » ${ }^{68}$. Au-delà de cette affirmation, il fait de cette période le point de départ «d'une escalade dans la confusion du droit de la guerre et des Droits de l'homme » ${ }^{69}$, notamment par l'adoption de ladite résolution 2675 (XXV), une confusion qui serait dommageable pour le droit des conflits armés, voire pour la résolution desdits conflits ${ }^{70}$.

Mais loin d'un processus d'amalgame entre ces ensembles normatifs, cette possible application simultanée et concurrente des Droits de l'homme et du droit humanitaire met en lumière, dans un premier temps, l'existence d'un fonds commun de normes ${ }^{71}$, énoncées autour d'un but unique. La prise en compte des droits fondamentaux dans les conflits armés montre le lien qui existe entre la DUDH et les principes nouveaux du droit international humanitaire. Ainsi, à la première conférence d'experts sur le développement des normes de droit humanitaire de 1971, convoquée par le CICR pour rédiger un projet de protocole additionnel aux Conventions de Genève, les membres ont pu affirmer que les Droits de l'homme et le droit humanitaire ont un but commun, à savoir assurer une véritable protection de la personne humaine et de ses droits fondamentaux ${ }^{72}$. Toutefois, pour réaliser cette protection, "international humanitarian law devotes special attention to the characteristics of the armed conflict and to the fact that, in these situations, the normal conditions of life in the nation are completely modified ${ }^{73}$. Les experts en concluent donc que toutes les évolutions et le renforcement de ce corpus normatif contribuent à la protection des droits fondamentaux de la personne humaine sans qu'il soit besoin de faire directement référence au droit des Droits de l'homme. Pourtant, comme le souligne Marc Schreiber, représentant du secrétaire général des Nations unies à la $2^{\mathrm{e}}$ session tenue un an après, «[à] la première session de la Conférence, nombre d'experts gouvernementaux se sont référés au fait que ces règles [des Droits de l'homme] trouvent leur application non seulement en temps de paix mais également en période de conflit armé et, notamment, en cas de conflit armé ne présentant pas un caractère international ${ }^{74}$. Afin de résoudre le problème qui se pose alors,

65. Assemblée générale des Nations unies, Résolution 2675 (XXV). Principes fondamentaux touchant la protection des populations...

66. Une vision confirmée par le secrétaire général des Nations unies, Rapport du secrétaire général au Conseil de sécurité sur la protection des civils en période de conflit armé, 8 septembre 1999, UN Doc. S/1999/957, \$3: «Le droit international humanitaire et les instruments juridiques relatifs aux Droits de l'homme énoncent les droits des civils et les obligations des combattants en période de conflit. »

67. H. Meyrowitz, «Le droit de la guerre et les Droits de l'homme», p. 1069-1070.

68. Ibid., p. 1061.

69. Ibid., p. 1069

70. Major M.A. Hansen, «Preventing the Emasculation of Warfare: Halting the Expansion of Human Rights Law into Armed Conflict ", Military Law Review, vol. 194, 2007. Pour ce juriste du Judge Advocate General étatsunien, cette confusion «could make winning wars nearly unachievable for those who try to comply with its strict requirements, and "[e]xcessive' humanization might exceed the limits acceptable to armed forces, provoke their resistance, and thus erode the credibility of the rules". Furthermore, humanization also could serve to unnecessarily prolong armed conflict, and thereby increase the evils of war that it purports to eradicate. Therefore, the unconstrained expansion of human rights law into matters of war must be stopped, for the safe of Soldiers and humanity alike». Cette vision n'est malheureusement pas sans rappeler une célèbre pensée de F. Nietzsche, Par-delà le bien et le mal, Paris, Union générale d'édition, 1970, $\$ 146$, p. 103: «Quand on lutte contre des monstres, il faut prendre garde de ne pas devenir monstre soi-même. Si tu plonges longuement ton regard dans l'abîme, l'abîme finit par ancrer son regard en toi. »

71. TPIY, Chambre d'appel, Celebici Case, arrêt, 20 février 2001, IT-96-21, $\$ 149$ : "The universal and regional human rights and the Geneva Conventions share a common "core" of fundamental standards which are applicable at all times, in all circumstances and to all parties, and from which no derogation is permitted. The object of the fundamental standards appearing in both bodies of law is the protection of the human person from certain heinous acts considered as unacceptable by all civilised nations in all circumstances."

72. CICR, Conference Of Government Experts On the Reaffirmation and Development of International Humanitarian Law Applicable in Armed Conflicts, Genève, 24 mai-12 juin 1971, vol. 1, document CE/1b, p. 30. Cf. également TPIY, Chambre de première instance, Le Procureur c. Anto Furundzija, jugement, 10 décembre 1998, IT-95-17/1, \$183: «Le principe général du respect de la dignité humaine est à la base du droit international humanitaire et des Droits de l'homme et en est, en fait, la raison d'être; il est désormais si important qu'il imprègne le droit international dans son ensemble »; TPIY, Chambre de première instance, Le Procureur c. Zlatko Aleksovski, jugement, 25 juin 1999, IT-95-14/1, $\$ 54$ : « on ne saurait concevoir de valeur plus importante que le respect de la personne humaine. On peut dire que tout l'édifice du droit international relatif aux Droits de l'homme et de l'évolution du droit international humanitaire repose sur ce principe fondamental»; TPIY, Chambre d'appel, Celebici Case, arrêt, 20 février 2001, IT-96-21, $\$ 149$; T. Meron, «International Law in the Age of Human Rights. General Course on Public International Law », RCADI, t. 301,2003 , p. 30 : « The fact that the law of war and human rights law have different historical and doctrinal roots has not prevented the principle of humanity from becoming the common denominator of both systems."

73. CICR, Conference Of Government Experts On the Reaffirmation and Development of International Humanitarian Law Applicable in Armed Conflicts, vol. 1, document CE/1b, p. 31

74. CICR, Conférence d'experts gouvernementaux sur la réaffirmation et le développement du droit international humanitaire applicable dans les conflits armés, seconde session, 3 mai-3 juin 1972, Rapport sur les travaux de la conférence, vol. I, Genève, CICR, 1972, \$ 2.69, p. 71. 
Marc Schreiber propose « d'inclure dans le protocole une référence expresse au fait que le protocole s'applique sans préjudice des principes et règles consacrés par les instruments des Nations unies relatifs aux Droits de l'homme ${ }^{75}$. Plusieurs experts, sans déposer de proposition à ce sujet, se joignirent à cet avis, «étant donné que l'on ne peut faire abstraction, dans l'application du droit international, de ces instruments [DUDH, Pactes internationaux...] $»^{76}$. Mais si le protocole II rappelle en son préambule «que les instruments internationaux relatifs aux Droits de l'homme offrent à la personne humaine une protection fondamentale ${ }^{77}$, le protocole I ne fait qu'évoquer, dans sa section III concernant le traitement des personnes au pouvoir d'une partie au conflit, les «autres normes applicables du droit international qui régissent la protection des droits fondamentaux de l'homme pendant un conflit armé de caractère international ${ }^{78}$, laissant planer un doute sur le contenu réel de ces normes et une possible inclusion des Droits de l'homme au sein de ces «autres normes».

Au détour de son avis consultatif sur la Licéité de la menace ou de l'emploi d'armes nucléaires, la Cour internationale de justice a levé une partie du voile en affirmant que «la protection offerte par le pacte international relatif aux droits civils et politiques ne cesse pas en temps de guerre ${ }^{79}$, sauf dérogation possible au regard des dispositions de son article 4. En l'espèce, le droit à la vie, dont il était question, n'est pas susceptible de dérogation, et son respect vaut également pendant des hostilités. Ainsi, nul ne peut être arbitrairement privé de la vie en temps de paix ou de conflit armé. Toutefois, la Cour se fonde sur la doctrine de la lex specialis pour apprécier le caractère arbitraire de cette privation. La Cour fait donc du droit des Droits de l'homme la lex generalis à laquelle dérogerait « le droit applicable dans les conflits armés, conçu pour régir la conduite des hostilités ${ }^{80}$. En cas de conflit armé, ce ne serait donc qu'en fonction des normes de droit des conflits armés applicables que devrait être interprété le caractère arbitraire, au regard de l'article 6 du Pacte international relatif aux droits civils et politiques (PIDCP), du décès provoqué par l'emploi d'un certain type d'armes, en l'espèce les armes nucléaires ${ }^{81}$.
Cette approche de la doctrine de la lex specialis s'écarte de la vision traditionnellement mise en avant de cet adage. En effet, la CIJ n'écarte pas la règle des Droits de l'homme au profit du droit humanitaire mais elle interprète la portée du droit à la vie, tel que prévu par le PIDCP, au regard des normes régissant les conflits armés ${ }^{82}$. Elle semble suivre sur ce point certaines réflexions de la Conférence d'experts gouvernementaux de 1971. Ces experts établissent un lien effectif entre les Droits de l'homme et le droit humanitaire, soulignant notamment que " the provisions of the Geneva Conventions protecting prisoners of war when questioned by the enemy, or forbidding the latter to hold them in dangerous zones, may be attached to the general rule formulated in Article 7 of the Covenant on Civil and Political Rights forbidding cruel or inhumane treatment ${ }^{83}$. Mais ils précisent que, justement, les règles de droit humanitaire « extend beyond, detail, or develop certain of the rules set forth in the Covenants on human rights ${ }^{84}$, car ces derniers ne sont pas suffisamment détaillés pour envisager les situations spécifiques liées à un conflit armé. Ainsi, l'utilisation de cet adage interprétatif ne reflète pas un antagonisme existentiel entre les deux corpus normatifs. En effet, "ces adages et techniques [telle la lex specialis] n'opèrent pas mécaniquement, mais font office de "principes directeurs" en donnant à entendre qu'il existe un rapport pertinent entre les règles applicables, eu égard à la nécessaire cohérence de la conclusion avec les finalités ou fonctions, telles qu'elles sont perçues, du système juridique dans son ensemble ${ }^{85}$. De ces interactions entre le droit international des Droits de l'homme et le droit des conflits armés semble naître progressivement un système normatif cohérent, un nouveau cadre conceptuel.

\section{DUDH et conflits armés ou une approche systémique de la société internationale}

Dans son discours à l'Assemblée générale des Nations unies du 19 novembre 1998, le juge McDonald, alors président du Tribunal pénal international pour l'exYougoslavie, a qualifié les deux Tribunaux pénaux internationaux de «gardiens des droits tels qu'énoncés

75. Ibid.

76. Ibid., $\$ 2.539$, p. 122.

77. Protocole additionnel aux Conventions de Genève du 12 août 1949 relatif à la protection des victimes des conflits armés non internationaux (protocole II), 8 juin 1977, préambule, alinéa 2.

78. Protocole additionnel aux conventions de Genève du 12 août 1949 relatif à la protection des victimes des conflits armés internationaux (protocole I), 8 juin 1977 , article 72.

79. CIJ, Licéité de la menace ou de l'emploi d'armes nucléaires, avis consultatif, 8 juillet 1996, CIJ Rec., 1996, \$25, p. 225 sq.

80. Ibid.

81. Ibid.

82. Même s'il est vrai que l'on peut à bon droit critiquer le rôle marginal laissé au droit des Droits de l'homme dans cet avis consultatif. Voir V. GowlandDebbas, "The Right to Life and Genocide: The Court and International Public Policy ", in International Law: The International Court of Justice and Nuclear Weapons, L. Boisson de Chazournes et P. Sands (dir.), Cambridge, Cambridge University Press, 1999, p. 321-326.

83. CICR, Conference Of Government Experts On the Reaffirmation and Development of International Humanitarian Law Applicable in Armed Conflicts, vol. 1, document CE/1b, p. 30-31.

84. Ibid.

85. M. Koskenniemi, Fragmentation du droit international: difficultés découlant de la diversification et de l'expansion du droit international, Rapport du groupe d'étude de la Commission du droit international, 13 avril 2006, UN Doc. A/CN.4/L.682, \$36. 
dans la Déclaration universelle ${ }^{86}$. Cette citation nous rappelle le point d'ancrage du système international qui s'est mis en place après la seconde guerre mondiale. Le maillage de relations réciproques des débuts du droit international tend à trouver une assise commune. Les interactions entre les différentes branches laissent apparaitre une cohérence globale de l'ensemble normatif. Expression d'une coutume dont l'application en temps de conflit armé semble possible, la DUDH sert ainsi de cadre à l'apparition d'une société internationale ${ }^{87}$.

Cette étude des rapports entre la Déclaration de 1948 et les conflits armés nous a permis de montrer l'existence d'un but commun entre ces deux ensembles normatifs, un but qui reflétait celui d'une société internationale en germe dès 1918. Comprenant donc cette société internationale comme un système, c'est-à-dire « un ensemble d'éléments en interaction dynamique, organisé en fonction d'un but ${ }^{88}$, nous pouvons en appréhender l'évolution au regard des interactions entre les sous-ensembles qui la composent.

Primitivement lié au paradigme de la souveraineté étatique, «le comportement du système résult[ait] des interactions entre parties équipotentielles ${ }^{89}$, mais «progressivement [est apparue] la subordination à des parties dominantes ${ }^{90}$. Le régime principalement bilatéral de l'avant-guerre s'est vu progressivement supplanté par un multilatéralisme qui tend à la fragmentation ${ }^{91}$, voire à la complexité. Parallèlement, «la spécialisation progressive» du système s'est vue «étroitement connectée avec la centralisation progressive qui s'exprime par l'évolution en fonction d'un élément dominant ${ }^{92}$. Cette base d'analyse issue des théories de L. von Bertalanffy sur la systémique nous semble être un angle d'attaque intéressant pour mieux comprendre ce nouveau paradigme du droit international qui se dégage à partir des années 1970-1980.

Afin d'éclairer les choses, il convient ici de compléter notre analyse des rapports entre la DUDH et les conflits armés. Il nous faut reprendre là où nous nous étions arrêtés, à savoir l'idée d'une lex generalis, mais en mettant en lumière l'élément dominant centralisateur que sont, nous allons le voir, les droits indérogeables. $\mathrm{Si}$, comme le souligne Mireille Delmas-Marty, la Déclaration de 1948 ne parle pas de ces droits indérogeables, « [1]'article 29-2 de la Déclaration admet des "limitations", à la double condition qu'elles soient prévues par la loi et répondent aux exigences "du respect des droits et libertés d'autrui" ou de la morale, de l'ordre public et du bien-être général dans une société démocratique. C'est reconnaître la possibilité de conflits entre les droits d'une personne et ceux d'autrui ; c'est surtout ménager aux valeurs collectives de l'ordre généralement associées aux exigences politiques de la raison d'État, un espace de négociation, espace largement ouvert par les termes employés : la morale, l'ordre public et le bien-être général. La question se pose aussitôt de savoir si tous les droits énoncés sont soumis à de telles limitations ou si certains d'entre eux y échappent et bénéficient d'une protection absolue ${ }^{93}$.

Un début de réponse est apporté par l'Assemblée générale des Nations unies quand elle affirme qu' «une situation dans laquelle un État serait mêlé à un conflit armé peut donc justifier certaines restrictions temporaires apportées à l'exercice de certains des Droits de l'homme proclamés dans la Déclaration ${ }^{94}$. Toutefois, par définition, les restrictions n'ont pas la même portée que les dérogations. Ainsi, comme le rappelle R. Ergec, «les restrictions sont impuissantes à porter atteinte à la substance des droits garantis» alors que «la notion de dérogation comporte, en revanche, la possibilité d'une ingérence en principe libre de tout obstacle matériel ${ }^{95}$. Mais toujours selon cet auteur, la Déclaration universelle « contient en son article 29 une clause générale de limitation qui pourrait remplir les fonctions d'une clause dérogatoire ${ }^{96}$. Il semble en effet que Mireille DelmasMarty, en faisant référence à des droits bénéficiant d'une "protection absolue», s'est déplacée sur le terrain de la dérogation ${ }^{97}$.

86. G. K. McDonald, Président du TPIY, «Address to the United Nations General Assembly», 19 novembre 1998, disponible sur www.un.org/icty/ pressreal/SPE981119.htm (consulté le 26 juin 2008) : "In a sense, our Tribunal was created to fill the gap left by the drafters of the Universal Declaration. When the Security Council established the International Tribunal in 1993, no one could have predicted that within one year a second Tribunal-for Rwanda - would be necessary. The establishment and success of these Tribunals played no small role in the signing, in July, of the Rome Treaty, creating a permanent International Criminal Court. Together, these courts are the guardians of the rights as enshrined in the Universal Declaration.»

87. Dans sa thèse, Olivier de Frouville voit dans les Droits de l'homme le moteur d'une « expansion du droit de la Société humaine universelle au détriment du droit de la Société des États souverains ». O. de Frouville, L'intangibilité des Droits de l'homme en droit international, Paris, Pedone, 2004 , p. 48.

88. J. de Rosnay, Le macroscope, Paris, Seuil, 1975.

89. L. von Bertalanffy, Théorie générale des systèmes, p. 70.

90. Ibid.

91. Voir notamment P.-M. Dupuy, «The Danger of Fragmentation or Unification of the International Legal System and the International Court of Justice», p. 791 sq. ; G. Hafner, «Les risques que pose la fragmentation du droit international », Rapport de la Commission du droit international, 20oo, UN Doc. A/55/10, annexe, p. 305-322.

92. L. von Bertalanffy, Théorie générale des systèmes, p. 69.

93. M. Delmas-Marty, «Le crime contre l'humanité, les Droits de l'homme et l'irréductible humain », Revue de science criminelle et de droit pénal comparé, $\mathrm{n}^{\circ} 3,1994$, p. 485-486.

94. Assemblée générale des Nations unies, Le respect des Droits de l’homme en période de conflit armé, rapport du secrétaire général, 20 novembre 1969 , UN Doc. A/7720, $\$ 24$.

95. R. Ergec, Les Droits de l'homme à l'épreuve des circonstances exceptionnelles. Étude sur l'article 15 de la Convention européenne des Droits de l'homme, Bruxelles, Bruylant, 1987, p. 34.

96. Ibid., p. 222.

97. À la suite de Nicole Questiaux, Olivier de Frouville parle pour sa part de «droits absolument intangibles» et de «droits relativement intangibles ». Les premiers sont ceux qui ne supportent aucune atteinte, même en période exceptionnelle, tandis que les seconds accepteraient des limitations dans de telles 
Communément, on parle de droits indérogeables lorsque, quelles que soient les circonstances, aucune licence n'est laissée aux États d'en exclure l'application dans certaines hypothèses prévues à l'avance. Sont ainsi notamment compris les droits référencés aux articles 4 du PIDCP, 15 de la Convention européenne des Droits de l'homme $(\mathrm{CEDH})$ et 27 de la Convention interaméricaine des Droits de l'homme (CIADH). Comme le souligne justement Jean Salmon, il s'agit en fait de la prévision ou non «d'exceptions à la règle » ${ }^{98}$, par les États parties à la convention en cause, en cas de circonstances exceptionnelles. Il est toutefois important de comprendre que le principe de la lex specialis ne joue pas sur ce plan-là. Si la loi spéciale déroge à la loi générale, c'est dans le sens où elle substitue un régime normatif à un autre ${ }^{99}$, alors que l'exception correspond à «la restriction par un acte (législatif) unilatéral ${ }^{100}$. Nous ne nous situons donc pas, comme dans le cas de l'exception ou de la limitation, dans un rapport vertical entre une norme de droit international et un acte interne, mais dans un rapport horizontal de substitution d'une norme internationale à une autre. Au final, seule l'existence d'une norme de jus cogens interdit la mise en œuvre de ce principe de résolution des conflits normatifs. En effet, le jus cogens a «selon la conception classique, la fonction de neutraliser l'opération du principe lex specialis derogat legi generali en frappant de nullité l'accord contraire. Le jus cogens est donc le principe anti-dérogation par excellence; c'est l'antimatière de la dérogation ${ }^{101}$.

Mais le problème de la compréhension des rapports entre les Droits de l'homme et le droit des conflits armés se complique au regard de l'identité possible des cas ouvrant droit à exception ou à application du principe de la lex specialis, les conflits armés étant inclus dans l'ensemble plus large des circonstances exceptionnelles.

Pour reprendre le cas détaillé dans l'avis consultatif de la CIJ concernant la Licéité de la menace ou de l'emploi d'armes nucléaires, nous sommes en présence du droit à la vie, reconnu comme non susceptible d'exception au regard de l'article 4 du PIDCP. Si des circonstances exceptionnelles n'autorisent pas les États à porter temporairement atteinte à ce droit, une période de conflit armé devrait normalement entraîner l'applicabilité des normes de droit humanitaire réglementant cette question, en vertu du principe de la lex specialis. Comme le souligne la CIJ, cette période de conflit armé n'entraînerait pas l'inapplicabilité totale du PIDCP, mais la Cour aurait dû faire prévaloir l'application des normes de droit humanitaire en cas d'applicabilité concurrente entre normes des deux corpus, comme en l'espèce sur le droit à la vie. Il nous semble donc qu'il manque dans cette affaire un élément pour comprendre la jurisprudence de la CIJ. La seule "indérogeabilité » du droit à la vie au regard du PIDCP ne peut expliquer l'interprétation de l'article 6 du PIDCP au regard des normes du droit des conflits armés, et de fait, l'applicabilité de cet article du corpus des Droits de l'homme en cas de conflit armé.

Il est donc logique de penser que la CIJ a implicitement considéré le caractère de jus cogens de cette interdiction de la privation arbitraire de la vie telle qu'énoncée à l'article 6 du PIDCP, faisant passer ce droit à la vie d'un droit indérogeable à un droit intangible, à savoir un droit qui cumule donc au moins les caractéristiques d'indérogeabilité et de jus cogens. Toutefois, considérant une approche à notre sens systémique du droit international, la Cour a interprété le caractère arbitraire de la privation de la vie au regard des normes de droit humanitaire. Cette approche peut être qualifiée de systémique dans le sens où le souci de globalité du système prime sur l'individualité de la norme. Ainsi, "si la discontinuité exclut une subordination étroite entre différents ensembles normatifs, elle ne signifie pas pour autant leur autonomie absolue. Des relations s'instaurent dont la topologie reste à établir, ce terme évoquant la théorie mathématique, applicable aussi bien en géométrie qu'en algèbre, qui étudie les relations de voisinage entre les espaces apparemment discontinus ${ }^{102}$.

Quelques années plus tard, confrontée à de multiples violations de différents droits garantis par le PIDCP, la CIJ pourra préciser que: «[d]e manière plus générale, la Cour estime que la protection offerte par les conventions régissant les Droits de l'homme ne cesse pas en cas de conflit armé, si ce n'est par l'effet de clauses dérogatoires du type de celle figurant à l'article 4 du pacte international relatif aux droits civils et politiques. Dans les rapports entre droit international humanitaire et Droits de l'homme, trois situations peuvent dès lors se présenter: certains droits peuvent relever exclusivement du droit international humanitaire; d'autres peuvent relever exclusivement des Droits de l'homme; d'autres enfin peuvent relever à la fois de ces deux branches du droit international. Pour répondre à la question qui lui est posée, la Cour aura en l'espèce à prendre en considération les deux branches du droit international précitées, à savoir les Droits de l'homme et, en tant que lex specialis, le droit international humanitaire ${ }^{103}$. »

Omettant, il est vrai, de rappeler le caractère de lex specialis du droit humanitaire, elle renouvellera cette

circonstances. O. de Frouville, L'intangibilité des Droits de l'homme..., p. 70 ; Nicole Questiaux, Sous-Commission de la lutte contre les mesures discriminatoires et de la protection des minorités, Rapport sur les conséquences pour les Droits de l'homme des développements récents concernant les situations dites d'état de siège ou d'exception, 27 juillet 1982, UN Doc. E/CN.4/Sub.2/1982/15, p. 15.

98. Dictionnaire de Droit international public, J. Salmon (dir.), Bruxelles, Bruylant, 2001, p. 326.

99. R. Kolb, "Jus cogens, intangibilité, intransgressibilité, dérogation “positive” et "négatives" ", RGDIP, vol. 109, n² 2, $2005, \mathrm{p} .322$.

100. Ibid., p. 323 (note 47).

101. Ibid., p. 323 .

102. M. Delmas-Marty, Le flou du droit, p. 26. Ou, pour reprendre Paul Valéry, «celui qui se représente un arbre est forcé de se représenter un ciel ou un fond pour l'y voir s'y tenir ». P. Valéry, Introduction à la méthode de Léonard de Vinci, Paris, Éditions de la Nouvelle revue française, 1919 , p. 12.

103. CIJ, Conséquences juridiques de l'édification d’un mur dans le territoire palestinien occupé, avis consultatif, CIJ Rec., 2004, \$106, p. 136 sq. 
interprétation dans l'affaire des activités armées sur le territoire du Congo (République démocratique du Congo c. Ouganda) du 19 décembre $2005^{104}$.

Au regard de cette jurisprudence, il convient donc de répertorier les trois situations auxquelles la Cour fait référence. En cas de conflits armés, premièrement, ne relèvent exclusivement du corpus des Droits de l'homme que les droits ne se trouvant pas en « conflit » avec une norme plus spéciale du droit humanitaire ${ }^{105}$. Leur application se fait au regard des possibles clauses échappatoires prévues par les instruments internationaux. Deuxièmement, le schéma inverse vaut pour les situations relevant exclusivement du droit des conflits armés, soit parce que l'État a fait jouer une clause échappatoire dans l'application d'un instrument des Droits de l'homme, soit parce que la règle en cause est spécifique à une situation de conflit armé (par exemple, la réglementation sur la distinction entre civils et combattants).

Enfin, toute la question vient de l'application concurrente des deux corpus normatifs. Nous l'avons vu précédemment, une première réponse a été apportée par la CIJ pour les droits dits intangibles, à savoir une application du droit des Droits de l'homme en cause mais interprété, dans ses conditions de mise en œuvre, au regard des normes du droit des conflits armés ${ }^{106}$. Dans un autre cas, si les deux normes portant sur le même sujet ne sont pas en contradiction, on constate alors leur possible application simultanée ${ }^{107}$. Cette solution a, par exemple, été utilisée par la CIJ dans l'affaire des activités armées sur le territoire du Congo ${ }^{108}$ lorsqu'elle constate une violation à la fois de l'article 7 du PIDCP ${ }^{109}$ et de l'article 32 de la Convention de Genève IV de $1949{ }^{110}$. À l'inverse, si les deux normes se contredisent mais que la norme des Droits de l'homme n'est pas un droit intangible, on constate une application du principe de lex specialis, mais avec une interprétation de la norme humanitaire au regard du système général des Droits de l'homme. Ainsi, la Chambre de première instance du TPIY a pu juger dans l'affaire Celebici qu' : " [i]l serait en effet contraire à l'intention du Conseil de sécurité, dont le souci était de faire face effectivement à une situation qui, estimait-il, constituait une menace pour la paix et la sécurité internationales, et de mettre un terme aux souffrances de toutes les personnes prises dans le conflit, que le Tribunal international refuse à un groupe de personnes le bénéfice de la IV ${ }^{\mathrm{e}}$ Convention de Genève uniquement sur la base du droit interne de la nationalité $[. .$.$] . Cette interpréta-$ tion de la Convention va tout à fait dans le sens de l'évolution de la doctrine du droit humanitaire qui a pris une importance croissante au cours de ces cinquante dernières années. Il serait contraire au concept même de Droits de l'homme, qui protège les individus des abus de leur propre État, d'appliquer de façon rigide la condition de la nationalité posée par l'article 4, condition apparemment insérée pour empêcher toute immixtion dans les rapports entre un État et ses propres ressortissants. De surcroît, la nature du conflit armé international en BosnieHerzégovine reflète la complexité de nombreux conflits modernes et probablement pas le paradigme envisagé en 1949. Afin de préserver la pertinence et l'efficacité des normes des Conventions de Genève, il est nécessaire d'adopter l'approche choisie ici ${ }^{111}$.»

Face à un droit des Droits de l'homme applicable à toute personne sans distinction aucune dans son rapport à un État, le TPIY applique en l'espèce les catégories du droit des conflits armés, implicitement en tant que lex specialis, en les interprétant toutefois au regard du «concept même de Droits de l'homme».

Ce passage est symptomatique de la nécessité, ressentie, il nous semble, par les juridictions internationales d'une approche systémique de la société internationale ${ }^{112}$. Les termes utilisés par le TPIY de « complexité»

104. CIJ, Affaire des activités armées sur le territoire du Congo (République démocratique du Congo c. Ouganda) du 19 décembre 2005 , $\$ 216$.

105. Nous ne reviendrons pas ici sur l'applicabilité extraterritoriale des Droits de l'homme, une condition bien sûr nécessaire pour traiter des rapports entre Droits de l'homme et droit des conflits armés. Pour plus de détails sur cette question, voir notamment N. Lubell, «Challenges in Applying Human Rights Law to Armed Conflict", RICR, $\mathrm{n}^{\circ} 860$, décembre 2005, p. 739 ; CIJ, Conséquences juridiques de l'édification d’un mur dans le territoire palestinien occupé, avis consultatif, CIJ Rec., 2004, \$109-111.

106. La Cour européenne des Droits de l'homme semble développer une jurisprudence similaire sur cette question. Cf. notamment l'analyse de l'arrêt Issaieva c. Russie de 2005 par M. Divac Öberg, «Le suivi par le Conseil de l'Europe du conflit en Tchétchénie », Annuaire français de droit international, vol. 50, 2004, p. 761-762.

107. Cette possibilité a d'ailleurs été soulignée par la Commission des Droits de l'homme, Résolution 2005/63. Protection des droits fondamentaux des personnes civiles en temps de guerre, 20 avril 2005, UN Doc. E/CN.4/2005/L.11/Add.7, p. 42-44: "Les actes qui constituent une violation du droit international humanitaire, y compris les infractions graves des Conventions de Genève du 12 août 1949 ou du protocole additionnel aux Conventions de Genève relatif à la protection des victimes des conflits armés internationaux (protocole I) du 8 juin 1977, peuvent aussi constituer une violation flagrante des Droits de l'homme.»

108. CIJ, Affaire des activités armées sur le territoire du Congo (République démocratique du Congo c. Ouganda), arrêt, 19 décembre 2005 , \$219.

109. Pacte international relatif aux droits civils et politiques, Assemblée générale des Nations unies, résolution 2200 A (XXI), 16 décembre 1966, art. 7 : « Nul ne sera soumis à la torture ni à des peines ou traitements cruels, inhumains ou dégradants. En particulier, il est interdit de soumettre une personne sans son libre consentement à une expérience médicale ou scientifique.»

110. Convention (IV) de Genève relative à la protection des personnes civiles en temps de guerre, 12 août 1949, art. 32: «Les Hautes Parties contractantes s'interdisent expressément toute mesure de nature à causer soit des souffrances physiques, soit l'extermination des personnes protégées en leur pouvoir. Cette interdiction vise non seulement le meurtre, la torture, les peines corporelles, les mutilations et les expériences médicales ou scientifiques non nécessitées par le traitement médical d'une personne protégée, mais également toutes autres brutalités, qu'elles soient le fait d'agents civils ou d'agents militaires. »

111. TPIY, Chambre de première instance II, Le Procureur c. Zejnil Delalic, Zdravko Mucic alias «Pavo », Hazim Delic, Esad Landzo alias «Zenga», jugement, 16 novembre 1998, IT-96-21, \$263 et 266. Cf. également T. Meron, «International Law in the Age of Human Rights... », p. 26-27.

112. Si nous partageons avec P. Meyer-Bisch l'idée d'une approche systémique des ensembles normatifs, il nous semble dommage que cet auteur affirme que les Droits de l'homme constituent en soi « un système dont il faut achever la "clôture" » (cf. P. Meyer-Bisch, « Méthodologie pour une présentation 
et de "paradigme» reflètent ce souci d'unité du droit international remis en avant face à la fragmentation de cet ordre juridique. Il semble donc que l'on puisse trouver une cohérence globale dans l'approche jurisprudentielle des rapports entre les Droits de l'homme et le droit humanitaire en période de conflits armés ${ }^{113}$. Et, même s'il n'emploie pas expressément les termes de lex specialis, de complexité ou de paradigme, le Comité des Droits de l'homme semble faire une approche similaire de la question. Rappelant son observation générale $n^{\circ} 29^{114}$, il a ainsi affirmé que: "[1]e Pacte s'applique aussi dans les situations de conflit armé auxquelles les règles du droit international humanitaire sont applicables. Même si, pour certains droits consacrés par le Pacte, des règles plus spécifiques du droit international humanitaire peuvent être pertinentes aux fins de l'interprétation des droits consacrés par le Pacte, les deux domaines du droit sont complémentaires et ne s'excluent pas l'un l'autre ${ }^{115}$. »

La complémentarité affirmée et affichée entre ces deux ensembles normatifs ${ }^{116}$ se fonde donc sur des interactions réciproques, rendues possibles par un fonds commun de normes. Ces éléments, reflétant les interac- tions au sein d'une globalité, correspondent aux deux premiers éléments d'une approche systémique des rapports entre ces corpus. Ce que nous appellerons finalement la société internationale, ou le paradigme de la complexité, nécessite également de comprendre les buts de l'organisation qui lui donnent son sens, orientent ses processus, définissent ses moyens. Cette dimension téléologique est inhérente à tout système normatif.

Il n'est donc au final pas inutile de rappeler ici le but même des rédacteurs de la Déclaration de 1948 : à savoir, comme le souligne Eleanor Roosevelt en 1948, de rédiger « une affirmation des principes fondamentaux qui déterminent les droits imprescriptibles de l'homme destinée à établir la norme vers laquelle doivent tendre tous les peuples et toutes les nations ${ }^{117}$. Ainsi, pour paraphraser Mireille Delmas-Marty, la Déclaration universelle serait devenue « une manière de produire de la conscience juridique $[. .$.$] en indiquant, non pas le contenu de la règle$ de droit, mais le droit du droit, c'est-à-dire, la règle de jugement qui permet de tracer la limite entre ce qui est, ou ne peut pas être du droit ${ }^{118}$.

systémique des droits humains ", Classer les Droits de l'homme, E. Bribosia et L. Hennebel (dir.), Bruxelles, Bruylant, 2004, p. 47-85). Cette réduction de l'analyse aux seuls Droits de l'homme est, à notre sens, une erreur au regard de la complexité de l'ordre international.

113. A contrario, voir A. Guellali, «Lex specialis, droit international humanitaire et Droits de l'homme : leur interaction dans les nouveaux conflits armés », p. 544-547. Selon cette auteure, la CIJ aurait fait une appréciation circonstanciée, une " approche pragmatique ", du rapport entre le droit international humanitaire et le droit international des Droits de l'homme pour ne pas interdire l'utilisation des armes nucléaires. Elle en conclut que « [1]a lex specialis apparaît ainsi comme une règle technique utilisée par la Cour au cas par cas pour résoudre un conflit concret de normes, et non une prise de position théorique et absolue sur les relations générales entre droit international humanitaire et Droits de l'homme». Elle voit ensuite une évolution de la vision de la CIJ sur cette question. Ainsi, dans son avis sur les Conséquences juridiques de l'édification d'un mur dans le territoire palestinien occupé, la Cour ne privilégierait plus «le recours exclusif au droit humanitaire par rapport aux Droits de l'homme. Elle examine les dispositions des deux corps de normes de manière égale ». Enfin, récemment dans son arrêt République démocratique du Congo c. Ouganda, la CIJ omettait de rappeler le caractère de lex specialis du droit humanitaire pour ne plus garder que l'application commune des deux corpus de règle.

114. Comité des Droits de l’homme, Observation générale n²9: états d’urgence (article 4), 31 août 2001, UN Doc. CCPR/C/21/Rev.1/Add.11, \$3.

115. Comité des Droits de l'homme, Observation générale n 31 : La nature de l’obligation juridique générale imposée aux États parties au Pacte [Art. 80], 26 mai 2004, UN Doc. CCPR/C/21/Rev.1/Add.13, \$11.

116. Une complémentarité soulignée encore récemment par la Commission des Droits de l'homme, Résolution 2005/63. Protection des droits fondamentaux des personnes civiles en temps de guerre, 20 avril 2005, UN Doc. E/CN.4/2005/L.11/Add.7, p. 42-44: « Consciente que le droit des Droits de l'homme et le droit international humanitaire sont complémentaires et se renforcent l'un l'autre; Estimant que tous les Droits de l'homme nécessitent une protection égale et que la protection garantie par le droit des Droits de l'homme demeure dans les situations de conflit armé, eu égard aux circonstances dans lesquelles le droit international humanitaire s'applique en tant que lex specialis.»

117. Eleanor Roosevelt, Assemblée générale des Nations unies, $3^{\mathrm{e}}$ session, $3^{\mathrm{e}}$ commission, $89^{\mathrm{e}}$ séance, 30 septembre 1948, document A/C.3/SR.89.

118. M. Delmas-Marty, Le flou du droit, p. 348. 
